\title{
Diagnostic challenges in Salla disease
}

\author{
Jessica N. Hartley ${ }^{1,2}$, Michael S. Salman ${ }^{3,4}$, Frances A. Booth ${ }^{3,4}$, Lorne Seargeant ${ }^{3,5}$, \\ David A. Wenger ${ }^{6}$, Jens Wrogemann ${ }^{7}$, Aizeddin A. Mhanni ${ }^{1,2,3^{*}}$ \\ ${ }^{1}$ Department of Biochemistry and Medical Genetics, University of Manitoba, Winnipeg, Canada \\ ${ }^{2}$ Program of Genetics and Metabolism, Winnipeg, Canada \\ ${ }^{3}$ Department of Pediatrics and Child Health, University of Manitoba, Winnipeg, Canada \\ ${ }^{4}$ Section of Pediatric Neurology, University of Manitoba, Winnipeg, Canada \\ ${ }^{5}$ Diagnostic Services Manitoba, University of Manitoba, Winnipeg, Canada \\ ${ }^{6}$ Lysosomal Diseases Testing Laboratory, Thomas Jefferson University, Philadelphia, USA \\ ${ }^{7}$ Department of Radiology, Section of Pediatric Radiology, University of Manitoba, Winnipeg, Canada \\ Email: ${ }^{*}$ amhanni@hsc.mb.ca
}

Received 27 October 2012; revised 16 November 2012; accepted 9 December 2012

Copyright (C) 2013 Jessica N. Hartley et al. This is an open access article distributed under the Creative Commons Attribution License, which permits unrestricted use, distribution, and reproduction in any medium, provided the original work is properly cited.

\begin{abstract}
Sialic acid storage disease (Salla disease) is an autosomal recessive disorder caused by mutations in a lysosomal sialic acid export protein, SLC17A5 (OMIM \#604369). This disorder was initially described in Northern Finland but more recently has been reported in patients of other ethnicities. We describe the clinical presentation and the neuroimaging findings of two non-Finnish children where a diagnosis of Salla disease was suspected on the basis of brain magnetic resonance imaging. The biochemical confirmation of this diagnosis posed a challenge as both patients had elevated percent free urine sialic acid but biochemical analyses in fibroblasts were not conclusive; therefore, molecular testing was necessary for confirmation of the diagnosis. The described encounters demonstrate the importance of pursuing confirmatory molecular diagnostic testing when a sialic acid storage disorder is suspected.
\end{abstract}

Keywords: Sialic Acid; Salla Disease; Lysosomal; SLC17A5

\section{BACKGROUND}

Free sialic acid storage disease (Salla disease) is an autosomal recessive disease caused by mutations in a lysosomal sialic acid export protein, SLC17A5 (OMIM \#604369) which leads to lysosomal accumulation of free sialic acid [1]. Common features of this disease include developmental delay, ataxia, subtle coarse facial features and brain magnetic resonance imaging (MRI) findings of

${ }^{*}$ Corresponding author. hypomyelination and hypoplastic corpus callosum [2]. Biochemical findings of a defect in the sialic acid export protein include elevated total and free sialic acid in urine and fibroblasts $[3,4]$. The mild to moderate form of this condition was initially described in Northern Finland with the eponym "Salla" referring to the geographically restricted area where the first family resided [4]. Since that time, free sialic acid storage disease has been reported in patients of other ethnicities and infantile free sialic acid storage disease (ISSD) (OMIM\# 269920) has been described to be allelic to Salla, with ISSD being associated with an infantile onset progressive disease and extremely elevated total and free sialic acid levels $[1,3$, $5,6]$. We describe the clinical presentation, neuroimaging and the challenges in confirming this diagnosis in two children of non-Finnish ancestry.

\section{MATERIALS AND PATIENTS}

\subsection{Methods}

Urine Sialic Acid Measurements: Determinations of urine total and free sialic acid levels were conducted using a spectrophometric method as described $[7,8]$ and compared to age-matched controls [9].

Sialic Acid Studies in Fibroblasts: A pellet of cultured skin fibroblasts was sonicated in distilled water and the protein concentration was determined. The total sialic acid content were determined after heating an aliquot containing between 100 and $150 \mu \mathrm{g}$ protein at $80^{\circ} \mathrm{C}$ in $0.1 \mathrm{~N}$ sulfuric acid for 1 hour. The same size aliquot in distilled water and not heated was used to determine the free sialic acid content. The method of Aminoff was then followed to measure the sialic acid content [10]. After reading the absorbance at both 549 and $532 \mathrm{~nm}$, the sialic 
acid content per mg protein was calculated.

This case series has been reviewed by the University of Manitoba Research Ethics Board to ensure that patient identity is protected and consent processes were adhered to.

\subsection{Patients}

Patient 1 presented at age twenty-two months with a history of global developmental delay. The family history was unremarkable for neurodegenerative disease and there was no history of consanguinity. The family was of Irish, English, Ukrainian and Dutch descent (non-Finnish). The pregnancy history was unremarkable with no history of exposure to known teratogens. The perinatal and neonatal histories were unremarkable. His birth weight was $3.486 \mathrm{~kg}$ (75th percentile). His Apgar scores were 5 and 8 at 1 and 5 minutes respectively. His examination at twenty-two months of age was remarkable for subtle coarse facial features, a depressed nasal bridge, a high arched palate and a high forehead. His weight was $12.8 \mathrm{~kg}$ (50th - 75th percentile), height was $84.5 \mathrm{~cm}$ (25th - 50th percentile) and OFC was $50.5 \mathrm{~cm}$ (90th 97 th percenttile). Hepatomegaly was noted with a palpable liver edge $3 \mathrm{~cm}$ below the right costal margin in the midclavicular line. His neurologic examination revealed normal visual behaviour and extraocular movements and generalized hypotonia (central and peripheral) with normal muscle strength. His deep tendon reflexes were normal and symmetric in the upper limbs and slightly increased in the lower limbs. Ataxia was noted in the lower but not the upper limbs. A formal developmental assessment using the Yale Developmental Schedules at twentyseven months of age revealed a developmental quotient of $65 \%$. The child's ophthalmologic and echocardiographic assessments were unrevealing. An electroencephalogram revealed left-sided slowing in background activity and right-sided epileptiform discharges; however there was no history of clinical seizures. Brain MRI at age twenty-one months showed abnormalities suggestive of Salla disease (Figure 1).

Patient 2 presented at age thirty-one months with a history of ataxia and developmental delay. The pregnancy, perinatal, neonatal and family histories were unremarkable. No consanguinity was reported and the family was not of Finnish ancestry. The child's examination at thirty-one months of age was remarkable for subtle coarse facial features and significant ataxia. The weight was $14 \mathrm{~kg}$ (75th percentile), height was $93.5 \mathrm{~cm}$ (50th percentile) and OFC was $48.8 \mathrm{~cm}$ (75th - 90th percentile). Cranial nerve examination was remarkable for excessive drooling with an intact gag response. Muscle strength and tone were normal. The child had prominent truncal and limb ataxia affecting all four extremities and walked only with support with a broad-based gait. The child had a bilateral pincer grasp but had difficulty with fine finger

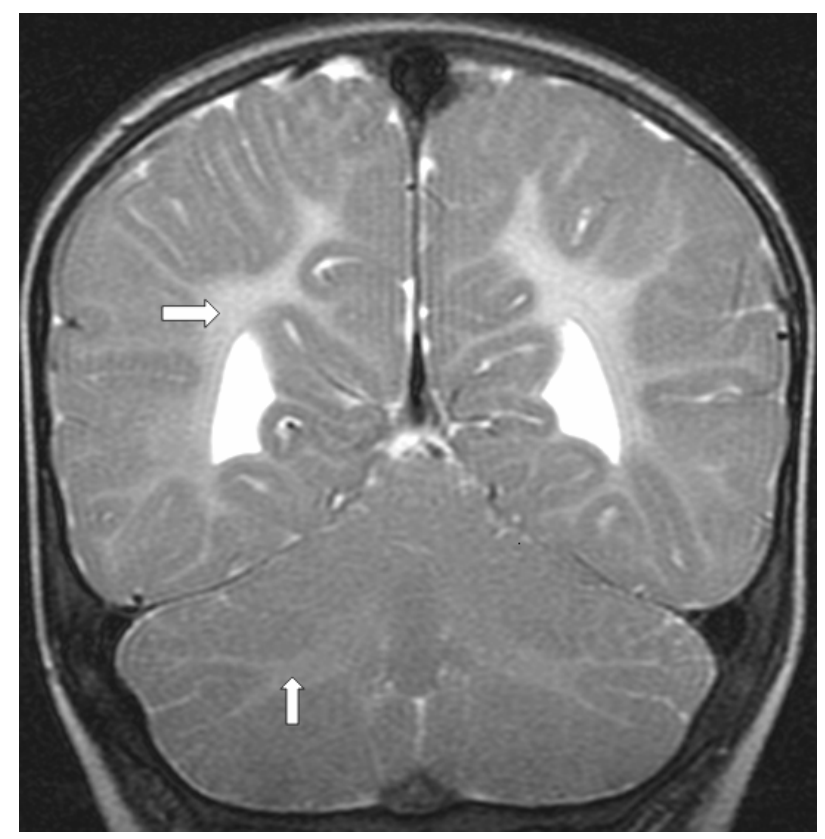

Figure 1. Coronal T2-weighted MRI in patient 1 at age twentyone months showing complete lack of normal myelin signal and white matter volume loss throughout the cerebral hemispheres (horizontal arrow). Hypomyelination and volume loss of the cerebellar white matter was also present (vertical arrow). The cortical gray matter and deep gray matter (not shown) were preserved.

movements and finger-to-nose testing. Deep tendon reflexes were normal and symmetric with down-going plantar responses. A formal developmental assessment using the Yale Developmental Schedules at thirty-one months of age revealed a developmental quotient of $65 \%$. Follow-up assessment using the Revised Gesell Developmental Schedules at sixty-nine months of age (five years) suggested a gross motor developmental quotient of $26 \%$, a fine motor developmental quotient of $50 \%$ and a language developmental quotient of $35 \%$. An electroencephalogram revealed localized epileptiform abnormalities. Brain MRI at age forty months also showed abnormalities suggestive of Salla disease (Figure 2).

\section{RESULTS}

A summary of the results of the laboratory investigations undertaken in both patients to investigate the possibility of Salla disease in provided in Table $\mathbf{1}$.

In patient 1 , urine total and free sialic acid were elevated above age-matched control values on two occasions, with the percent free urine sialic acid and skin electron microscopy being suggestive of a free sialic acid storage disorder (data not shown). Fibroblast assay revealed elevations of free sialic acid in the diagnostic range, however total sialic acid values were only mildly elevated as compared to controls. Molecular analysis of the SLC17A5 gene revealed compound heterozygosity 
Table 1. Pertinent laboratory investigations for Salla disease.

\begin{tabular}{|c|c|c|}
\hline & Patient 1 & Patient 2 \\
\hline Urine Average Free Sialic Acid (umol/mmol creatinine) & $313^{*}$ & 93.5 \\
\hline Urine Average Total Sialic Acid (umol/mmol creatinine) & $387^{*}$ & 146 \\
\hline $\begin{array}{l}\% \text { Urine Free Sialic Acid } \\
(\text { Salla }>80 \%) \\
(\text { Control }<60 \%)\end{array}$ & $80.9 \%{ }^{*}$ & $63.8 \%{ }^{*}$ \\
\hline \multicolumn{3}{|l|}{ Fibroblast Sialic Acid (nmol/mg protein) } \\
\hline $\begin{array}{c}\text { Free SA } \\
(\text { Salla } 10.0+/-2.9 \mathrm{SD})^{* *} \\
(\text { Control } 1.0+/-0.6 \mathrm{SD})\end{array}$ & $10.4^{*}$ & 2.1 \\
\hline $\begin{array}{c}\text { Total SA } \\
\text { (Control } 18.0+/-4.6 \mathrm{SD})\end{array}$ & 20 & 15.3 \\
\hline Skin Electron Microscopy & Lysosomal storage of fine fibrillar material & $\begin{array}{l}\text { Enlarged lysosomes with fine fibrillar } \\
\text { structures }\end{array}$ \\
\hline Molecular & $\begin{array}{l}\text { Pat c. } 250 \text { del C } \\
\text { Mat c. } 115 \mathrm{C}>\mathrm{T}\end{array}$ & Homozygous c. $115 \mathrm{C}>\mathrm{T}$ \\
\hline
\end{tabular}

"Diagnostic laboratory values; ${ }^{* *}$ Case/control values from Seppala et al. 1991.

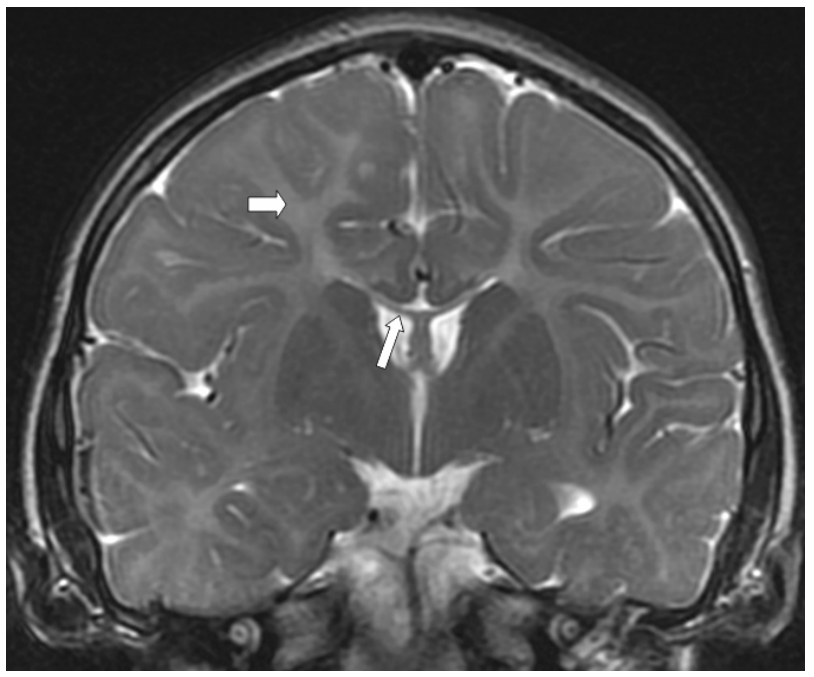

Figure 2. Coronal T2-weighted MRI in patient 2 at age forty months showing marked volume loss and increased T2 signal intensity of the cerebral hemispheric white matter (horizontal arrow) and corpus callosum (oblique arrow), reflecting a lack of myelin. The cortical gray matter and deep gray matter were preserved. There was no evidence of volume loss or signal abnormality in the posterior limbs of the internal capsule, cerebellum, and brainstem (not shown).

for the common Finnish mutation, 115C > T (R39C), and another mutation, 250delC, previously described in a French patient [11] confirming the diagnosis of Salla disease.

In patient 2, four independent urine samples revealed borderline elevations of free and total sialic acid. Urine percent free sialic acid was mildly elevated compared to age-matched control values, but not in the diagnostic range for Salla disease. Skin electron microscopy was consistent with a free sialic acid storage disorder (data not shown). Fibroblast assay showed normal total and free sialic acid. Molecular analysis of the SLC17A5 gene revealed homozygosity for the common Finnish mutation, $115 \mathrm{C}>\mathrm{T}$ (R39C), confirming a diagnosis of Salla disease.

\section{DISCUSSION}

The clinical phenotype of both patients is consistent with Salla disease, rather than infantile free sialic acid storage disease (ISSD) given that symptoms developed during the first year of life and that both patients continued to make developmental progress, albeit slowly, without any evidence of developmental regression [5]. The elevated total but not free sialic acid in fibroblast culture in patient one and the normal total and free sialic acid in fibroblast culture for patient two posed a diagnostic challenge. The more severe ISSD is associated with extremely high total and free sialic acid levels in cultured fibroblasts, leukocytes and urine as compared to the milder Salla disease [3]. To our knowledge, there are no published reports of individuals with phenotypic Salla disease with normal fibroblast free sialic acid, even among the Finnish population where the condition was originally described. A pair of siblings with a Salla phenotype and homozygosity for a different mutation, K136E, also had non-diagnostic biochemical findings, with normal urine free sialic acid (percent free sialic acid not reported), elevated sialic acid in cerebrospinal fluid and mildly elevated free sialic acid in fibroblasts [12]. Normal leukocyte total and free sialic acid levels in mildly affected children of Mennonite descent, who are homozygous for the R39C Finnish mutation, like patient 2, have been noted. In the urine of these patients, the total sialic acid content was noted to be normal, however the percent free was elevated above normal (greater than $80 \%$ free versus less than $60 \%$ in non-Salla individuals) 
(Wenger, personal communication 2012). Our patient two was comparable, with mildly elevated urine free sialic acid, however, $63 \%$ free sialic acid is not considered to be in the diagnostic range.

Molecular diagnostic testing was required for confirmation of the diagnosis in both our patients as well as those noted by Wenger (personal communication 2012) and reported by Mochel [12]. Patient 2 was homozygous for the Finnish mutation associated with the Salla disease phenotype. It is interesting to note that neither of our patients is of Finnish ancestry but the R39C mutation is present in both. Salla disease due to the originally described R39C mutation appears to be pan-ethnic; homozygosity for this mutation has been reported in individuals of other ethnicities, including those of Old Order Mennonite extraction [1].

Our report demonstrates the importance not only by considering the diagnosis of Salla disease in children with subtle coarse facial features, developmental delay and hypomyelination on brain MRI who are not of Finnish ancestry, but also of the importance of pursuing molecular testing for Salla disease for confirmation of the diagnosis in the face of non-diagnostic biochemical analyses.

\section{REFERENCES}

[1] Aminoff, D. (1961) Methods for the quantitative estimation of $\mathrm{N}$-acetylneuraminic acid and their application to hydrolysates of sialomucoids. Biochemical Journal, 81, 384-392.

[2] Aula, P., Autio, S., Raivio, K., Rapola, J., Thoden, C., Koskela, S. and Yamashina, I. (1979) "Salla Disease": A new lysosomal storage disorder. Archives of Neurology, 36, 88-94. doi:10.1001/archneur.1979.00500380058006

[3] Aula, N., Salomaki, P., Timonen, R., Verheijen, F., Mancini, G., Mansson, J., Aula, P. and Peltonen, L. (2000) The spectrum of SLC17A5 gene mutations resulting in free sialic acid-storage diseases indicates some genotypephenotype correlation. American Journal of Human Ge- netics, 67, 832-840. doi:10.1086/303077

[4] Kelly, T.E. and Graetz, G. (1977) Isolated acid neuraminidase deficiency: A distinct lysosomal storage disease. American Journal of Medical Genetics, 1, 31-46. doi:10.1002/ajmg.1320010105

[5] Mochel, F., Yang, B., Barritault, J., Thompson, J., Engelke, U., McNeill, N., Benko, W., Kaneski, C., Adams, D., Tsokos, M., Abu-Asab, M., Huizing, M., Seguin, F., Wevers, R., Ding, J., Verheijen, F. and Schiffmann, R. (2009) Free sialic acid storage disease without sialuria. Annals of Neurology, 65, 753-757. doi:10.1002/ana.21624

[6] Romppanen, J. and Mononen, I. (1995) Age-related reference values for urinary excretion of sialic acid and deoxysialic acid: Application to diagnosis of storage disorders of free sialic acid. Clinical Chemistry, 41, 544-547.

[7] Seppala, R., Tietze, F., Krasnewich, D., Weiss, P., Ashwell, G., Barsh, G., Thomas, G., Packman, S. and Gahl, W. (1991) Sialic acid metabolism in sialuria fibroblasts. Journal of Biological Chemistry, 266, 7456-7461.

[8] Sonninen, P., Autti, T., Varho, T., Hämäläinen, M. and Raininko, R. (1999) Brain involvement in Salla disease. American Journal of Neuroradiology, 20, 433-443.

[9] Strauss, K., Puffenberger, E., Craig, D., Panganiban, C., Lee, A., Hu-Lince, D., Stephan, D. and Morton, D. (2005) Genome-wide SNP arrays as a diagnostic tool: Clinical description, genetic mapping, and molecular characterization of Salla disease in an Old Order Mennonite population. American Journal of Medical Genetics, 138A, 262-267. doi:10.1002/ajmg.a.30961

[10] Stephenson, R., Lubinsky, M., Taylor, H., Wenger, D., Schroer, R. and Olmstead, P. (1983) Sialic acid storage disease with sialuria: Clinical and biochemical features in the severe infantile type. Pediatrics, 72, 441-449.

[11] Verheijen, F., Verbeek, E., Aula, N., Beerens, C., Havelaar, A., Joosse, M., Peltonen, L., Aula, P., van der Spek, P. and Mancini, G. (1999) A new gene, encoding an anion transporter, is mutated in sialic acid storage diseases. Nature Genetics, 23, 462-465. doi:10.1038/70585

[12] Warren, L. (1959) The thiobarbituric acid assay of sialic acids. Journal of Biological Chemistry, 234, 1971-1975.

\section{ONLINE DATABASES CITED}

Online Mendelian Inheritance in Man, OMIM ${ }^{\circledR}$. Johns Hopkins University, Baltimore, MD. MIM Number: 604369: 03/07/2012. World Wide Web URL:

http://omim.org/

Online Mendelian Inheritance in Man, OMIM ${ }^{\circledR}$. Johns Hopkins University, Baltimore, MD. MIM Number: 269920: 07/22/2010. World Wide Web URL:

http://omim.org/ 